\title{
CRESCIMENTO INICIAL DE PLANTAS DE CAMU-CAMU SOB FERTIRRIGAÇÃO COM POTÁSSIO EM CONDIÇÕES DE TERRA FIRME
}

Carlos ABANTO-RODRÍGUEZ ${ }^{1}$, Edvan ALVES-CHAGAS ${ }^{3}$, Teresinha COSTA-SILVEIRA de ALBUQUERQUE ${ }^{3}$, Aline das GRAÇAS-SOUZA ${ }^{3}$, Wellington FARIAS-ARAÚJO², Pollyana CARDOSO$\mathrm{CHAGAS}^{4}$, Jeysse Kelly CARVALHO-de ANDRADE ${ }^{2}$, Olisson MESQUITA-SOUZA ${ }^{2}$

1 Instituto de Investigaciones da Amazônia Peruana, IIAP. Programa em Manejo Integral do Bosque e Serviços Ambientais (PROBOSQUES). Estação Experimental Dale E. Bandy Ucayali. Estrada Federico Basadre Km. 12.400, Pucallpa-Peru. Email: cabanto@iiap.org.pe,

2 Universidade Federal de Roraima (UFRR), Programa de Pós-graduação (POSAGRO)- Centro Ciências Agrárias-CCA (Campus Cauamé) BR 174, Km 12 - Monte Cristo wellington@cca.ufrr.br, jeyssekelly@hotmail.com, mesquita_ox@yahoo.com.br,

3 Embrapa Roraima -Rodovia BR-174, Km 8 Distrito Industrial Boa Vista, RR - Brasil - CEP 69301-970, E-mail: edvan.chagas@embrapa.br, teresinha.albuquerque@embrapa.br, alineufla@hotmail.com,

4 Escola Agrotécnica da Universidade Federal de Roraima Campus Murupu: Rodovia BR 174, km 35, s/n -P.A Nova Amazônia-69.300-000-BoaVista/RR.E-mail: pollyana.chagas@ufrr.br.

\section{RESUMO}

Uma das tendências do mundo moderno é a orientação ao consumo de produtos naturais com alto valor nutritivo e vitamínico. O camu-camu é uma fruteira nativa da Amazônia, que chama a atenção pelo alto conteúdo de vitamina $\mathrm{C}(6,116 \mathrm{mg} / 100 \mathrm{~g}$ de polpa). La espécie está em processo de domesticação, pelo qual se vem desenvolvendo modelos tecnológicos para lograr seu estabelecimento em condições de terra firme. Com o objetivo de avaliar diferentes doses de potássio aplicadas via fertirrigação no desenvolvimento inicial de plantas de camu-camu, conduziu-se um experimento no centro experimental Agua Boa da Embrapa Roraima. Utilizou-se o delineamento experimental em blocos casualizados com 5 tratamentos $0 ; 40 ; 80 ; 120$ e $160 \mathrm{~kg} \mathrm{ha}^{-1}$ de $\mathrm{K}$, aplicado em forma de $\mathrm{KCl}$, seis repetições e sete plantas por parcela experimental. $\mathrm{O}$ experimento foi conduzido durante 270 dias após o transplantío (DAT). A cada 30 dias foram avaliados o diâmetro do coleto $(\mathrm{mm})$ e altura de planta $(\mathrm{cm})$; logo a cada 90 dias foram avaliados o número de ramos e comprimento total de ramos $(\mathrm{cm})$. Ao final do experimento foi avaliado a massa seca das folhas, massa seca dos ramos, massa seca do sistema radicular, massa seca total (g) e o índice de qualidade de Dickson. Diante dos resultados, verificou-se que a fertilização potássica na dose $160 \mathrm{~kg} \mathrm{ha}^{-1}$ de $\mathrm{K}_{2} \mathrm{O}$ via fertirrigação proporcionou maior crescimento e qualidade de plantas camu-camu no primeiro ano de adubação em condições de terra firme.

PALAVRAS-CHAVE: Myrciaria dubia, Nutrição mineral, Nutrientes, Irrigação.

\section{EARLY GROWTH OF CAMU-CAMU PLANTS UNDER FERTIGATION WITH POTASSIUM UNDER SOILID GROUND CONDITIONS}

\section{ABSTRACT}

One of the trends of the modern world is the orientation to the consumption of natural products with a high nutritive and vitamin value. The camu-camu is a fruit-bearing tree native to the Amazon rainforest, which calls attention for its high vitamin $\mathrm{C}$ content $(6.116 \mathrm{mg} / 100 \mathrm{~g}$ of pulp). With the objective of evaluating different doses of potassium applied via fertigation on the early development of camu-camu plants, an experiment was conducted in the Água Boa Experimental Center of Embrapa Roraima. The experimental design in randomized blocks with 5 treatments $0 ; 40 ; 80 ; 120$ and $160 \mathrm{~kg}$ ha- 1 of $\mathrm{K}$ applied as $\mathrm{KCl}$, six replications and seven plants per experimental plot was utilized. The experiment was conducted for 270 days after transplanting (DAT). Every 30 days were evaluated the stem diameter $(\mathrm{mm})$ and plant height $(\mathrm{cm})$; every 90 days were evaluated the number of twigs and total length of twigs $(\mathrm{cm})$. At the end of the experiment were evaluated the a chlorophyll content, the dry matter of leaves (g), dry matter of twigs (g), dry mass of the root system (g), total dry mass (g), the Dickson quality index and the content and accumulation of macro and micronutrients. On the basis of the results, it has been found, that potassium fertilization at the dose $160 \mathrm{~kg} \mathrm{ha}^{-1}$ $\mathrm{K}_{2} \mathrm{O}$ through fertigation promoted higher growth and quality of plants camu-camu in the first year of fertilization under soilid ground conditions.

KEYWORDS: Myrciaria dubia, Mineral nutrition, Nutrients, Irrigation. 


\section{INTRODUÇÃO}

O camu-camuzeiro é uma espécie que tem despertado o interesse de produtores e consumidores, por possuir alto teor de vitamina $\mathrm{C}$, que chega a atingir até $6,116 \mathrm{mg} / 100 \mathrm{~g}$ de polpa. É uma fruteira de clima tropical úmido, que cresce nas várzeas que sofrem inundação periodicamente, dos rios e lagos de toda a bacia Amazônica (Esashika et al., 2011; Yuyama, 2002; Yuyama et al., 2011; Carvalho, 2012; Santos et al., 2009; Grigio, 2013; Abanto et al., 2014). A produção e o beneficiamento de seus frutos é uma alternativa viável ao desenvolvimento regional do camu-camu, como fonte de alimento e obtenção de renda das comunidades rurais e, além disso, favorece a preservação da espécie (Welter et al., 2011; Pinedo etal., 2001).

A produção de frutos em populações naturais não garante uma continua disponibilidade do produto em vista da sazonalidade ocasionada pelos diversos fatores edafoclimáticos. Dessa forma, torna-se imprescindível o cultivo em terra firme, mediante um manejo agronômico adequado com o uso de tecnologias para proporcionar maiores condições de desenvolvimento e produção.

Em Roraima existem boas perspectivas para o desenvolvimento do camu-camu em terra firme, no entanto por não ser uma espécie domesticada, pouco se sabe sobre as condições de manejo agronômico para o desenvolvimento inicial das plantas, este gera dificuldade na transferência de tecnologias, por não existir critérios definidos para a recomendação segura de adubação nos primeiros anos de cultivo.

Rodrigues et al. (2000) caracterizaram os solos da região de cerrado de Roraima como latossolos e argissolos amarelos pré-intemperizados, com limitações à produção de alimentos, por apresentarem baixa fertilidade natural e pela existência de uma estação seca bem definida, com duração de sete meses. Embora os princípios gerais de disponibilidade de nutrientes no solo sejam bastante conhecidos, sua aplicação em fruticultura enfrenta algumas dificuldades, pois ainda não existem critérios definidos para a recomendação segura de adubação em plantas nativas. O conhecimento das necessidades nutricionais da planta, bem como sua resposta à aplicação de fertilizantes, é fundamental para otimizar a eficiência de utilização destes, garantindo plantas vigorosas em curto espaço de tempo, além disso, as plantas adubadas corretamente atingem rapidamente, a altura e o diâmetro de coleto adequados à enxertia ou poda de formação em fruteiras (Serrano et al., 2004).
Entre os nutrientes indispensáveis para o crescimento e desenvolvimento das plantas destacase o potássio $\left(\mathrm{K}^{+}\right)$, que é um nutriente com diversos papéis no metabolismo vegetal. Atua como ativador enzimático de processos responsáveis pela síntese e degradação de compostos orgânicos e participa no processo de abertura e fechamento das células estomáticas, síntese de proteínas, osmorregulação, extensão celular e balanço entre cátions e ânions, além disso, é o nutriente requerido em larga quantidade pelas culturas, sendo o cátion mais abundante nos vegetais (Malavolta et al., 1989; Cretton, 2006; Albuquerque et al., 2009).

O suprimento inadequado de potássio ocasiona o funcionamento irregular dos estômatos, podendo diminuir a assimilação de $\mathrm{CO}_{2}$, e a taxa fotossintética (Cecílio e Grangeiro, 2004; Sampaio et al., 2005; Montoya et al., 2006). Por outro lado, aplicações excessivas do nutriente podem inibir a absorção de $\mathrm{Ca}^{2+} \mathrm{e} \mathrm{Mg}^{2+}$, bem como a diminuição na assimilação do fósforo, chegando, muitas vezes, a causar a deficiência desses nutrientes, resultando em efeitos depressivos sobre a produção das plantas (Pinto et al., 1994; Fortaleza et al., 2005; Silveira e Malavolta, 2006;).

A fertirrigação é a prática de aplicar fertilizantes dissolvidos na água de irrigação de forma contínua ou intermitente, permitindo aplicar o $\mathrm{K}$ diretamente na região de maior concentração de raízes das plantas e em doses fracionadas para o aumento na eficiência da adubação, fornecendo, além disso, a quantidade de água que a planta precisa (Teixeira et al., 2007; Andrade, 2004; Mantovani et al., 2003). Diante do exposto, este estudo teve por objetivo avaliar os componentes do crescimento das plantas de camu-camu, em função de doses de K, aplicadas via fertirrigação em condições de terra firme na região do cerrado de Roraima-Brasil.

\section{MATERIALE MÉTODOS}

A pesquisa foi realizada no período de janeiro a outubro do ano 2013, em uma área total de $2.464 \mathrm{~m}^{2}$, no Campo Experimental de Água Boa pertencente a EMBRAPA Roraima, situado nas coordenadas geográficas de $02^{\circ} 39^{\prime} 48,94^{\prime \prime}$ de latitude norte e $60^{\circ}$ $50^{\prime} 30,39^{\prime \prime}$ de longitude oeste, com uma altitude 90 $\mathrm{m}$ no Município de Boa Vista-Roraima. O clima da região é caracterizado como Aw-Tropical com estação seca, segundo a classificação de Köppen, com precipitação média de $1700 \mathrm{~mm}$ ano $^{-1}$ concentrada nos meses de abril a setembro e temperatura média anual entre 26 e $28{ }^{\circ} \mathrm{C}$. À evapotrasnpiração máxima mensal segundo o método de Blaney-Criddle é de 141,2 mm no mês de 
janeiro e à mínima é $103 \mathrm{~mm}$ para o mês de junho e utilizando o método de tanque classe A é de 147,3 mm no mês de janeiro (Araújo et al., 2007).

O solo da área do experimento é classificado como Latossolo Amarelo e apresenta os atributos químicos mostrados na Tabela 1 , realizado no laboratório de análises do Instituto Nacional de Pesquisa da Amazônia (INPA) conforme a metodologia da Embrapa-1998.

A área destinada à implantação do experimento com camu-camuzeiro foi previamente preparada, tendo sido roçada, arada e gradeada, para que todos os restos de biomassa vegetal existente na camada superficial do solo fossem incorporados. Devido o solo apresentar baixo $\mathrm{pH}$ e reduzidos teores de $\mathrm{P}, \mathrm{Ca}$ e $\mathrm{Mg}$, com elevada saturação de $\mathrm{Al}$ e baixa saturação de bases, realizou-se uma correção do solo em $\mathrm{Ca}$, $\mathrm{Mg}$ e $\mathrm{P}$, aplicando a lanço, após o preparo da área, $1.700 \mathrm{~kg} \mathrm{ha}^{-1}$ de calcário dolomítico e $400 \mathrm{~kg} \mathrm{ha}^{-1}$ de Superfosfato Simples (SS). No preparo das covas com dimensões de $0,40 \mathrm{~m} \times 0,40 \mathrm{~m} \times 0,40 \mathrm{~m}$, aplicou-se mais $500 \mathrm{~g}$ de calcário segundo as recomendações de Yuyama et al. (2011); e Itauran et al. (2002) e $50 \mathrm{~g}$ de SS/cova. Ao mesmo tempo foram adicionados $10 \mathrm{~g}$ de FTE-BR12 com a finalidade de enriquecer o solo com micronutrientes, visto que este produto apresenta a seguinte composição: 9,0 a $9,2 \%$ de $\mathrm{Zn} ; 1,8$ a $2,17 \%$ de $\mathrm{B}$; $0,80 \%$ de $\mathrm{Cu} ; 3,82 \%$ de Fe; 2,0 a $3,4 \%$ de $\mathrm{Mn}$ e $0,132 \%$ de Mo.

As mudas de camu-camu utilizadas no experimento foram provenientes de plântulas oriundas da população Candeias do banco de germoplasma do Instituo Nacional de Pesquisas da Amazônia - INPA. Essa população se caracteriza por apresentar arbustos menores de 2,5 $\mathrm{m}$ de altura, com copa laxa a densa (copa intermediária), com tronco ramificado a partir do solo ou a $20 \mathrm{~cm}$ do chão (Yuyama, 2011). As plântulas foram transplantadas para sacos contendo $1 \mathrm{dm}^{3}$ de substrato composto por areia, solo e serragem na proporção 1:1:1 em maio do 2012. Quando as mudas estavam com sete meses e apresentavam cerca de 3,7 ramos, com altura média de $35,6 \mathrm{~cm}$ e 4,4 $\mathrm{mm}$ de diâmetro de coleto foram transplantadas para as covas que haviam sido preparadas 25 dias antes.

Aos 135 DAT foi realizada uma segunda fertilização com $50 \mathrm{~g}$ de SS conforme recomendação de Yuyama (2011). Para suprir as exigências de N se adicionou uma dose padrão de $40 \mathrm{~kg} \mathrm{ha}^{-1}$ de ureia, em todos os tratamentos desde o inicio do experimento.

A irrigação foi realizada por sistema de gotejamento autocompensado acionado por motobomba ligada a uma caixa de água, com ativação automática do sistema mediante um programador de controle RAIN BIRD ${ }^{(\mathrm{R})}$ (“timer”). A vazão do sistema foi linear de $6,81 \mathrm{~h}^{-1}\left(3,41 \mathrm{~h}^{-1}\right.$ por cada gotejador espaçados a cada $50 \mathrm{~cm}$ ). Para a injeção dos fertilizantes utilizou-se o Injetor tipo Venturi de $3 / 4$ de polegada, operando com taxa de injeção de 150 litros/hora.

A quantidade de água aplicada foi determinada com base na evapotranspiração de referência estimada pelo tanque Classe A e o coeficiente da cultura (Kc) tomado como referencia, da goiabeira $(\mathrm{Kc}=4,5)$ indicado para o primeiro ano (Allen et al., 1998; Teixeira, 2003).

O delineamento experimental foi em blocos ao acaso com cinco tratamentos, que constaram das seguintes doses de potássio na forma de $\mathrm{KCl} \mathrm{em} \mathrm{kg}$ ha $^{-1}$ : T1-0; T2- 40; T3-80; T4-120 e T5-160 com seis repetições. Os tratamentos foram aplicados via fertirrigação durante 40 semanas, e as doses foram parceladas a cada 10 semanas perfazendo $10 \%$; $20 \% ; 30 \%$; e $40 \%$ do total da dose correspondente. A cultura do camu-camu foi implantada utilizando o espaçamento de $4 \mathrm{~m}$ entre fileiras e de $0,5 \mathrm{~m}$ entre plantas, sendo a parcela experimental constituída por sete plantas (cinco plantas úteis e duas de bordadura, só por condições do experimento).

Foram realizadas avaliações a cada 30 dias medindo-se a altura das plantas $(\mathrm{cm})$ e diâmetro do coleto $(\mathrm{mm})$ e a cada 90 dias foram avaliados o número de ramos e comprimento total dos ramos $(\mathrm{cm})$ por planta (estas variáveis foram analisadas no esquema de parcelas subdivididas no tempo, constituindo as parcelas as cinco doses e as subparcelas o tempo, sendo10 avaliações no tempo para altura e diâmetro, e quatro avaliações no tempo para número e comprimento de ramos). Aos 270 DAT no final do experimento, as plantas foram coletadas, separando-se as raízes da parte aérea (ramos e folhas), que foram colocadas para secar em estufa de circulação de ar forçada a $65^{\circ} \mathrm{C}$, até peso constante. Posteriormente avaliou-se a massa seca das amostras em gramas (Variáveis foram analisadas num delineamento em blocos ao acaso simples).

Com os resultados de massa seca da parte aérea (MSPA), massa seca das raízes (MSRZ) e massa seca total (MST), altura (H) e diâmetro do coleto (DC), determinou-se o índice de qualidade de Dickson (IQD) utilizando a fórmula descrita por Gomes et al. (2003), definida pela expressão $\mathrm{IQD}=\mathrm{MST} /[(\mathrm{H} / \mathrm{DC})+(\mathrm{MSPA} / \mathrm{MSRZ})]$.

Os dados foram submetidos à análise de variância e à regressão polinomial $(\mathrm{p}<0,05)$ pelo programa computacional SISVAR (Ferreira, 2010). Para valores que não estavam dentro dos padrões de normalidade e homogeneidade foram transformados $\mathrm{em}(\mathrm{x}+0,5)^{0,5}$. 


\section{RESULTADOS EDISCUSSÃO}

\section{Altura da planta $(\mathrm{cm})$, diâmetro do coleto $(\mathrm{mm})$, número e comprimento de ramos $(\mathrm{cm})$}

De acordo com a análise de variância para as características de crescimento das plantas de camucamu, não houve influência significativa pelo teste de $\mathrm{F}(\mathrm{p}<0,05)$ das doses de $\mathrm{K}$ para a altura $(\mathrm{cm})$, no entanto, foi significativa para o diâmetro do coleto (mm). A interação do tempo de aplicação e das doses de $\mathrm{K}$ foi significativa para as variáveis estudadas no período de 270 dias após o plantio (Tabela 2).

De acordo com a análise de variância para as características: número e comprimento $(\mathrm{cm})$ de ramos foram influenciados significativamente $(p<0,05)$, pelas doses de $K$, tempo de avaliação e pela interação dos dois fatores (Tabela 3 ).

As plantas de camu-camu ao receberem fertilização potássica apresentaram uma resposta linear para o número de ramos e quadrática para altura de plantas, comprimento dos ramos e diâmetro do coleto (Figura 1). Os tratamentos começaram a fazer efeito aproximadamente aos 25 dias após a primeira fertirrigação das mudas. Não houve resposta significativa $(p<0,05)$ da altura das plantas (Figura 1a) obtendo-se uma média de 105, $41 \mathrm{~cm}$ nas doses de potássio no final do experimento.

No entanto, o aumento linear na altura das plantas como resposta ao uso de doses crescentes de $\mathrm{K}$ tem sido observado em diferentes espécies por diversos autores: Marcussi et al. (2004), trabalhando com pimentão; Lima et al. (2001) trabalhando com adubação potássica em plantas de acerola. Ao analisar a Figura 1; observa-se influência das doses de potássio utilizadas sobre diâmetro do coleto ( $\mathrm{mm}$ ), número e comprimento de ramos conferindose o efeito significativo da dose de $160 \mathrm{~kg} \mathrm{ha}^{-1}$ de $\mathrm{K}$ nestas variáveis. No entanto, não foi atingido o ponto de máxima utilização deste nutriente, não havendo desta forma, um efeito depressivo do potássio sobre o crescimento das plantas.

Para o diâmetro do coleto (Figura 1b), as diferenças significativas $(p<0,05)$ entre os tratamentos foram observadas a partir dos 180 DAT, aproximadamente; sendo que as doses mais altas de $160 \mathrm{e} \mathrm{de} 120 \mathrm{~kg} \mathrm{ha}^{-1}$ as que apresentaram maior efeito positivo, obtendo-se um diâmetro médio de coleto de $15,78 \mathrm{~mm}$ e de $15,72 \mathrm{~mm}$, respectivamente, comparadas com o diâmetro de coleto de $10,9 \mathrm{~mm}$ obtido na testemunha.

Para a altura (Figura 1a) e diâmetro do coleto (Figura 1b), foram observados valores máximos de $105,41 \mathrm{~cm}$ e $15,78 \mathrm{~mm}$ em resposta as doses $160 \mathrm{~kg}$ $\mathrm{ha}^{-1}$ de $\mathrm{N}$, respectivamente, aos 270 DAT quando poderia ser realizada a primeira poda de formação das plantas, visto que estes resultados estão em conformidade com os valores encontrados por Abanto et al. (2011), que verificaram ser necessário que as plantas atinjam uma altura de 100 a $150 \mathrm{~cm}$ e 8 a $10 \mathrm{~mm}$ de diâmetro do coleto para que as plantas recebam este trato cultural.

As doses de $\mathrm{K}$ e as épocas de avaliação influenciaram significativamente o número de ramos e comprimento dos ramos das plantas de camu-camu, apresentando um modelo de resposta linear para o número de ramos e quadrático para seu comprimento (Figura 1c e 1d). Verificou-se que o maior número de ramos está associado à dose de 160 $\mathrm{kg} \mathrm{ha}^{-1}$, alcançando 29 ramos, o que representa um ganho em média de 10 ramos por planta, quando comparada com a testemunha (Figura 1c). Em relação ao comprimento de ramos (Figura 1d) a dose $160 \mathrm{~kg} \mathrm{ha}{ }^{-1}$ também influenciou nos melhores resultados obtendo-se em média $402,86 \mathrm{~cm}$ o que gerou aumento de $107,78 \%$ no tamanho dos ramos em relação a testemunha. Estes resultados indicam que as plantas de camu-camu, nessa dose de $\mathrm{K}$, apresentaram melhor estrutura, a qual fornecerá uma melhor copa na primeira poda formação, obtendo-se maior qualidade e quantidade de ramos para a frutificação.

No presente estudo as plantas de camu-camu que não receberam potássio tiveram seu crescimento comprometido, evidenciado pelo efeito significativo do K sobre a emissão de novas brotações e maior comprimento destas, o que demonstrou ser indispensável no início do plantio. Nesse sentido, Cecílio e Grangeiro (2004), Sampaio et al. (2005), Montoya et al. (2006) e Viana et al. (2007) citam que o suprimento inadequado de potássio ocasiona o funcionamento irregular dos estômatos, podendo diminuir a assimilação de $\mathrm{CO}_{2}$ e a taxa fotossintética, atrasando o crescimento das plantas em campo.

Malavolta e Romero (1975) descrevem como sintomas de plantas deficientes em K: tendência das folhas dobrarem-se para baixo e, em geral, são pouco desenvolvidas apresentando internódios mais curtos. Cakmak (2004) salientou que, em muitos casos, a expansão celular é consequência da acumulação de $\mathrm{K}$ nas células, necessária para estabilizar o $\mathrm{pH}$ do citoplasma e aumentar o potencial osmótico nos vacúolos, contribuindo para a elongação celuAlar.

\section{Massa de matéria seca $(\mathrm{g})$ das folhas, ramos, raízes e massa da matéria seca (g) total}

A aplicação de $\mathrm{K}$ via fertirrigação, promoveu efeitos significativos pelo teste $F(p<0,05)$ nas características de massa (g) seca da folha (MSFO), 
ramos (MSR), raiz (MSRZ) e massa (g) seca total (MST) (Tabela 4).

A resposta em biomassa de matéria seca (MSFO, MSR, MSRZ e MST) das plantas de camu-camu ao uso de doses crescentes de $\mathrm{K}$ aplicadas via fertirrigação no solo, apresentou um comportamento linear significativo $(\mathrm{p}<0,05)$, conforme se observa na Figura 2, indicando que as doses maiores não limitaram seus acréscimos. A dose de $160 \mathrm{~kg} \mathrm{ha}^{-1} \mathrm{de}$ $\mathrm{K}_{2} \mathrm{O}$ proporcionou um valor máximo de $302,69 \mathrm{~g}$ de MST; 203,69 g de MSR; 52,44 g de MSFO e 49,85 g MSRZ o que promoveu um ganho de $139,56 \%, 183,49 \%, 221,32 \%$ e $69,73 \%$, respectivamente em relação ao tratamento das plantas que não receberam adubação potássica (Figura 2).

Resultados similares foram observados por Natale et al. (2006), trabalhando com adubação potássica em plantas de maracujazeiro amarelo, obtendo respostas lineares com doses crescentes de $\mathrm{K}$.

Assim, estes resultados foram satisfatórios para o estudo, já que concordam com Souza et al. (2007) e Mendonça et al. (2009), os quais asseguraram, que a massa de matéria seca total é um acertado indicador para demonstrar o efeito do fertilizante sobre o crescimento das plantas. Por outro lado, Farinelli et al. (2003) observaram que a cultura do arroz apresentava boa produção com a aplicação da dose de nitrogênio de $100 \mathrm{~kg} \mathrm{ha}^{-1}$ sem adubação potássica. Entretanto, quando o potássio foi fornecido em uma dose baixa de $25 \mathrm{~kg} \mathrm{ha}^{-1}$, a dose ótima de nitrogênio $\left(100 \mathrm{~kg} \mathrm{ha}^{-1}\right)$ caiu para a metade, clara demonstração da importância do K para o equilíbrio nutricional e bom funcionamento fisiológico das plantas.

\section{Índice de qualidade de Dickson (IOD)}

$O$ índice de qualidade de Dickson (IQD) é apontado como bom indicador da qualidade das plantas, por considerar para o seu cálculo a robustez e o equilíbrio da distribuição da biomassa, sendo ponderadas várias características importantes (Gomes et al., 2003; Soares et al., 2007). Este índice é tradicionalmente utilizado na produção de mudas de espécies florestais, mas as mesmas características que compõe o IQD são importantes para a produção de mudas frutíferas (Dias et al., 2012). Nesse sentido quanto maior é o IQD melhor é a qualidade da planta, onde o valor 0,20 representa o valor mínimo (Gomes e Paiva, 2004). O IQD para o presente estudo apresentou um comportamento crescente linear em função das doses de $\mathrm{K}$. A dose que promoveu o índice máximo de qualidade de Dickson foi a $160 \mathrm{~kg}$ $\mathrm{ha}^{-1}$ de K reportando um IQD de 27,66 indicando que a qualidade de plantas aumentou com as doses crescentes de K (Figura 3).

Resultados semelhantes foram verificados por Welter (2011) com resposta linear crescente no IQD, trabalhando na produção de mudas de camu-camu com doses de pó de basalto. Do mesmo modo, Viana (2007) conferiu que a adubação potássica em trigo aumentou a qualidade de toda a planta devido ao aumento da eficiência da fotossíntese, ao aumento da resistência a algumas doenças e a ótima eficiência no uso da água.

Tabela 1. Atributos químicos do solo do campo experimental Água Boa da Embrapa Roraima, onde foi conduzido o experimento (2013).

\begin{tabular}{|c|c|c|c|c|c|c|c|c|c|c|c|}
\hline $\mathrm{pH}$ & MO*** & $\mathbf{P}^{* *}$ & $\mathbf{K}^{*}$ & $\mathrm{Ca}^{*}$ & Mg* & $\mathbf{H}+\mathbf{A l}$ & SB & CTC & Sat bases & Sat Al & $\mathbf{S}$ \\
\hline $\mathrm{CaCl}_{2}$ & $\mathrm{~g} \mathrm{dm}^{-3}$ & $\mathrm{~g} \mathrm{dm}^{-3}$ & \multicolumn{6}{|c|}{ - $\mathrm{mmol}_{\mathrm{c}} \mathrm{dm}^{-3}$} & V\% & $\mathrm{m} \%$ & $\mathrm{mg} \mathrm{dm}{ }^{-3}$ \\
\hline 4,4 & 10 & 3 & 0,2 & 2 & 1 & 24 & 3 & 28 & 11 & 72 & 6 \\
\hline \multicolumn{12}{|c|}{ Método: EDTA*, Mehlich1***, Método volumétrico***. } \\
\hline \multicolumn{3}{|c|}{$\mathrm{Cu}^{*}$} & \multicolumn{2}{|l|}{$\mathbf{Z n}^{*}$} & \multicolumn{2}{|c|}{$\mathrm{Fe}^{*}$} & \multicolumn{2}{|r|}{$\mathbf{B}^{* *}$} & \multicolumn{3}{|c|}{$M n^{*}$} \\
\hline \multicolumn{12}{|c|}{ - } \\
\hline \multicolumn{3}{|c|}{0,2} & \multicolumn{2}{|c|}{0,3} & \multicolumn{2}{|c|}{21} & \multicolumn{2}{|c|}{0,13} & \multicolumn{3}{|c|}{0,4} \\
\hline
\end{tabular}

* Método DTPA*; Método da Água quente**. 
Tabela 2. Análise de variância (ANOVA) do efeito do $\mathrm{K}$ sobre altura $(\mathrm{cm})$ e diâmetro do coleto $(\mathrm{mm})$ das plantas de camu-camu no período de 270 dias.

\begin{tabular}{clllcc}
\hline \multirow{2}{*}{ Fator de variação } & \multirow{2}{*}{ GL } & \multicolumn{1}{c}{ S0 } & OM & S0 & OM \\
\cline { 3 - 6 } & & Altura da planta & (cm) & Diâmetro do coleto & (mm) \\
\hline Doses (D) & 4 & 16,74 & $4,18^{\text {ns }}$ & 2,57 & $0,64^{*}$ \\
Erro 1 & 20 & 197,08 & 9,85 & 4,91 & 0,25 \\
Tempo (T) & 9 & 216,940 & $240,99^{*}$ & 314,66 & $34,96^{*}$ \\
T X D & 36 & 36,33 & $1,09^{*}$ & 8,54 & $0,24^{*}$ \\
Erro 2 & 1425 & 1030,98 & 0,72 & 100,737 & 0,07 \\
\hline Total & 1494 & $1.467,87$ & & 431.42 & \\
\hline CV (\%) 1 & & 40,52 & & 17,52 & \\
CV (\%) 2 & & 10,98 & & 9,39 & \\
\hline
\end{tabular}

* Significativo a 0,05\% de probabilidade pelo Teste $\mathrm{F}$. ${ }^{\text {ns }}$ Não significativo.

Tabela 3. Análise de variância (ANOVA) do efeito das doses de K sobre o número de ramos e comprimento $(\mathrm{cm})$ de ramos das plantas de camu-camu.

\begin{tabular}{llllll}
\hline \multirow{2}{*}{ Fator de variação } & \multirow{2}{*}{ GL } & \multicolumn{1}{c}{ S0 } & QM & \multicolumn{1}{c}{ S0 } & OM \\
\cline { 3 - 6 } & & Altura da planta & (cm) & Diâmetro do coleto & (mm) \\
\hline Doses (D) & 4 & 15,39 & $3,85^{*}$ & 380,42 & $95,10^{*}$ \\
\hline Erro 1 & 20 & 23,92 & 1,19 & 374,91 & 18,74 \\
\hline Tempo (T) & 3 & 685,27 & $228,42^{*}$ & 15228,96 & $5076,32^{*}$ \\
T X D & 12 & 21,25 & $1,77^{*}$ & 449,06 & $37,42^{*}$ \\
Erro 2 & 555 & 265,61 & 0,47 & 4042,08 & 7,28 \\
\hline Total & 594 & $1.011,44$ & & $20.475,43$ & \\
\hline CV (\%) 1 & & 32,24 & & 42,32 & \\
CV (\%) 2 & & 20,46 & & 26,38 & \\
\hline
\end{tabular}

* Significativo a 0,05\% de probabilidade pelo Teste $\mathrm{F}$. ${ }^{\text {ns }}$ Não significativo.

Tabela 4. Resumo da análise de variância (ANOVA) do efeito das doses de K sobre a massa (g) seca de folha (MSFO), massa seca de ramos (MSR), massa seca de raiz (MSRZ) e massa (g) seca total (MST) das plantas de camu-camu no período de 270 dias.

\begin{tabular}{clllll}
\hline \multirow{2}{*}{ Fator de variação } & \multirow{2}{*}{ GL } & \multicolumn{4}{c}{ Quadrados Médios } \\
\cline { 3 - 6 } & & \multicolumn{1}{c}{ MSF0 } & MSR & MSRZ & MST \\
\hline Doses & 4 & $1670,95^{*}$ & $23131,83^{*}$ & $634,89^{*}$ & $47180,29^{*}$ \\
Bloco & 5 & 99,36 & 1516,89 & 15,26 & 2468,48 \\
\hline Resíduo & 20 & 67,11 & 1058,65 & 36,92 & 1970,56 \\
\hline CV (\%): & & 23,24 & 22,95 & 15,07 & 20,42 \\
\hline
\end{tabular}

*-Significativo a $0,05 \%$ de probabilidade, pelo Teste $\mathrm{F}$. 

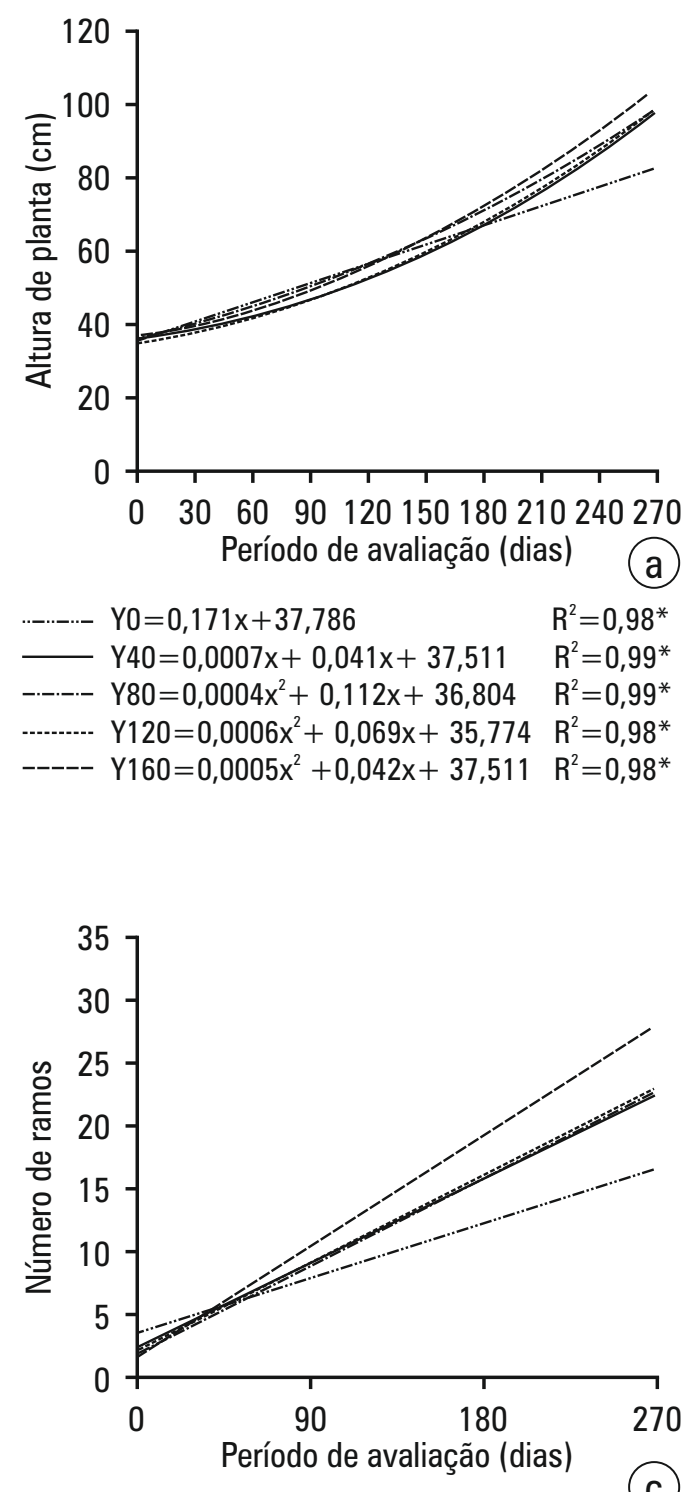

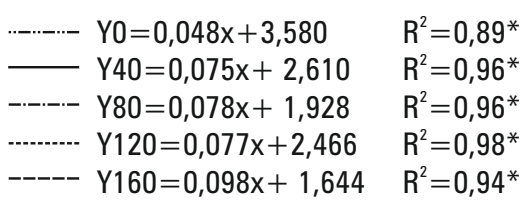

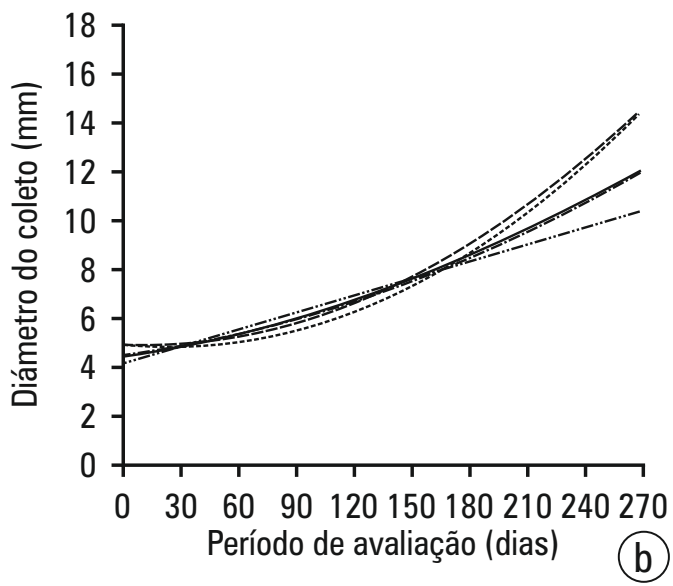

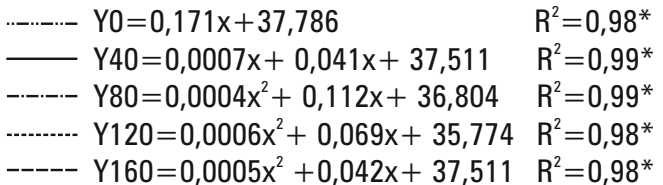

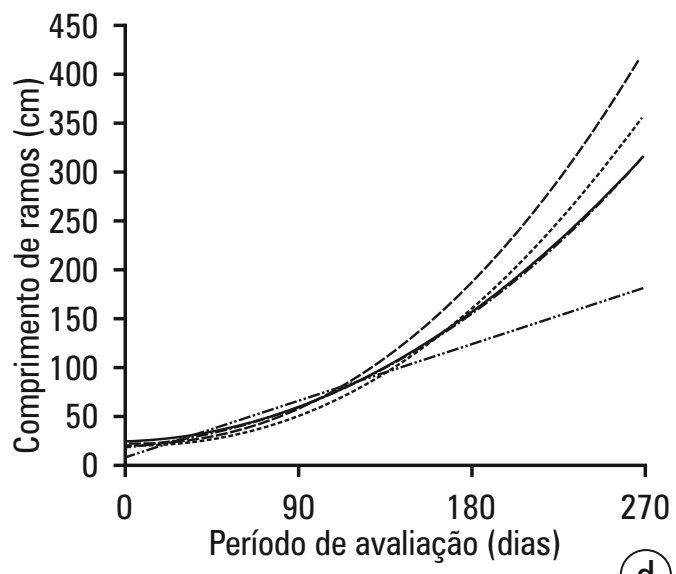

$$
\begin{array}{lc}
-\cdots-Y 0=0,659 x+5,212 & R^{2}=0,92^{*} \\
-Y 40=0,004 x^{2}+0,080 x+17,986 & R^{2}=0,98^{*} \\
-\cdots Y 8=0,004 x^{2}+0,062 x+21,133 & R^{2}=0,98^{*} \\
-\cdots 120=0,005 x^{2}+0,214 x+22,648 & R^{2}=0,99^{*} \\
-\cdots-Y 160=0,006 x^{2}-0,199 x+19,216 & R^{2}=0,99^{*}
\end{array}
$$

Figura 1. Efeito das doses de $\mathrm{K}$ sobre: a) altura $(\mathrm{cm})$; b) diâmetro do coleto $(\mathrm{mm})$; c) número; d) comprimento (cm) de ramos de plantas de camu-camu. 


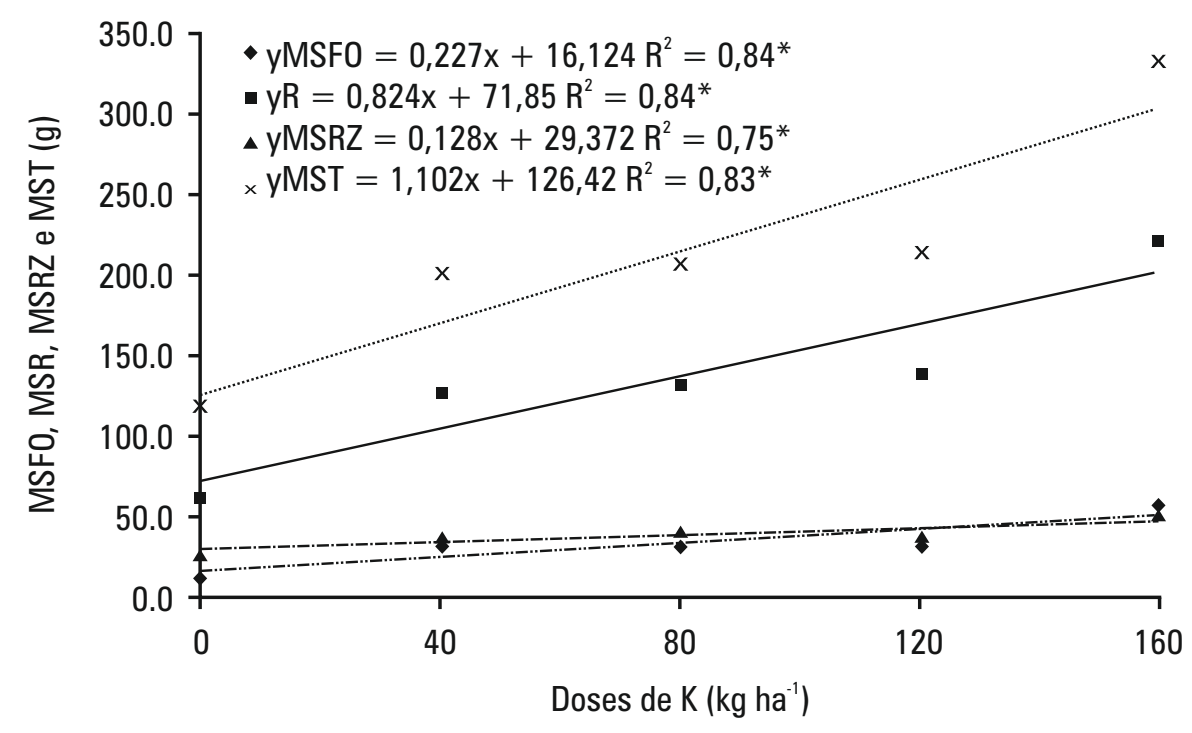

Figura 2. Efeito das doses de K sobre a massa (g) seca de folha (MSFO), de ramos (MSR), de raiz (MSRZ) e massa (g) seca total (MST) das plantas de camu-camu.

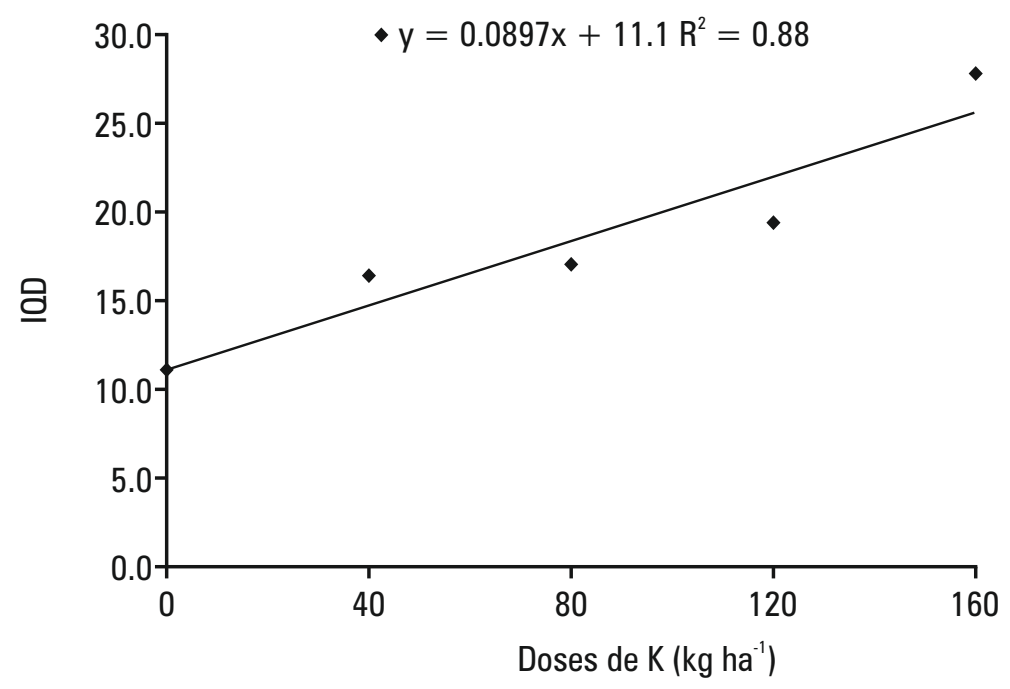

Figura 3. Efeito das doses de K sobre Índice de qualidade de Dickson (IOD) das plantas de camu-camu. 


\section{CONCLUSÕES}

A utilização da fertilização potássica aplicada mediante sistema de fertirrigação proporcionou maior qualidade nas plantas de camu-camu na dose de $160 \mathrm{~kg} \mathrm{ha}^{-1}$ de $\mathrm{K}$ na forma de $\mathrm{KCl}$, apresentando efeito positivo na altura de planta $(\mathrm{cm})$, diâmetro do coleto ( $\mathrm{mm}$ ), número e comprimento total dos ramos (cm), massa seca $(\mathrm{g})$ de raízes, ramos, folhas, massa seca total $(\mathrm{g})$ e índice de qualidade de Dickson.

Não foi atingindo o ponto de máxima eficiência nas variáveis estudadas devido ao não houver um balanço com a dose aplicada de $\mathrm{N}$ o que limitou o uso do $\mathrm{K}$ pela planta ou a quantidade de $\mathrm{K}$ fornecido as plantas foi insuficiente.

O parcelamento a cada 10 semanas de $10 \% ; 20 \%$; $30 \%$ e $40 \%$ e a utilização da fertirrigação possibilitou a maior absorção dos nutrientes pelas plantas de camu-camu em condições de terra firme.

A aplicação de $10 \mathrm{~g}$ de FTE BR 12 em cada cova de plantio foi suficiente para suprir a quantidade necessária de micronutrientes, para que as plantas sejam suficientemente nutridas durante o primeiro ano de cultivo.

É fundamental para o sucesso da fertilização que haja balanceamento entre os macronutrientes e os micronutrientes, pois ambos são essenciais para o bom crescimento das plantas de camu-camu.

\section{AGRADECIMENTOS}

Os autores desejam expressar seu agradecimento a CAPES, CNPq e Embrapa Roraima pelo auxílio financeiro do presente trabalho e a Universidade Federal de Roraima-Brasil pelo valioso aporte para a formação académica do autor principal.

\section{REVISÃO BIBLIOGRAFICA}

Abanto, C.R.; Chagas, E.A.; Choy, S. J.; Andrade dos Santos, V.; Lozano, R.M.B.; Ríos, G.S. 2014. Capacidad de enraizamiento de plantas matrices promisorias de Myrciaria dubia (Kunth) Mc Vaugh en cámaras de subirrigación. Revista Ceres 61(1): 134-140. doi: 10.1590/S0034$737 \mathrm{X} 2014000100018$

Abanto, C.; Choy, J.S.; Saldaña, W.; Paifa, M.; Chagas, E.A. 2011. Efecto de la altura de poda de formación en la arquitectura de plantas de camu-camu (Myrciaria dubia H.B.K. Mc Vaugh) en la estación experimental del IIAP, Ucayali, Perú. Scientia Agropecuaria, 2(2): 73 -81.

Andrade, B.M. 2004. Efeito da fertirrigação com nitrogênio e potássio sobre os teores de nutrientes em um solo cultivado com gravioleira. Revista Ciência Agronômica, 35(2): 410-417.

Araújo, F.W.; Costa, A.A.S.; Santos, E.A. 2007. Comparação entre métodos de estimativa da evapotranspiração de referência (ETO). Revista Caatinga, 20(4): 84-88.

Bugarín-Montoya, R.; Galvis-Spinola, A.; SánchezGarcia, P.; García-Paredes, D. 2006. Demanda de potasio del tomate tipo sadette. Terra Latinoamericana, 20(4): 391-399. (http://www.chapingo.mx/terra/contenido/20/4/ art391-399.pdf). Acesso: 04/01/2014.

Carvalho, A.S. 2012. Ocorrência, distribuição geográfica e estudo fenológico de camucamuzeiro (Myrciaria dubia (H.B.K.) Mc Vaugh) no estado de Roraima. Dissertação (Mestrado em Agronomia), Universidade Federal de Roraima, Boa Vista, Brasil. 79pp.

Cecílio Filho, A.B; Grangeiro, C.L. 2004. Produtividade da cultura da melancia em função de fontes e doses de potássio. Ciência $e$ Agrotecnologia, 28(3): 561-569. doi: 10.1590/S1413-70542004000300011

Cretton, V.C. 2006. Adubação potássica para a goiabeira (Psidium guajava L.) em formação na região norte fluminense. Dissertação (Mestrado em Produção Vegetal), Universidade Estadual Norte Fluminense, Campos dos Goytacazes, Brasil. 70pp.

Dias, M.J.T.; Souza, H.A.; Natale, W.; Modesto, V.C.; Rozane, D.E. 2012. Adubação com nitrogênio e potássio em mudas de goiabeira em viveiro comercial. Semina: Ciências Agrárias, 33 (6): 2837-2848.

Esashika, T.; Oliveira, L.A.; Moreira, F.W. 2011. Teores foliares de nutrientes em plantas de camucamuzeiro (Myrciaria dubia (H.B.K.) McVaugh) submetidas a adubações orgânica, mineral e foliar. Revista Brasileira de Ciências Agrárias, 6(3): 391-400.

Fortaleza, J.M.; Peixoto, J.R.; Junqueira, N.T.V.; Oliveira A.T. Rangel, L.E.P. 2005. Características físicas e químicas em nove genótipos de maracujá-azedo cultivado sob três níveis de adubação potássica. Revista Brasileira de Fruticultura, 27(1): 124-127. doi: 10.1590/S0100-29452005000100033

Gomes, J.M.; Couto, L.; Leite, H.G.; Xavier, A.; Garcia, S.L. R. 2003. Parâmetros morfológicos na avaliação da qualidade de mudas de Eucalyptus grandis, Revista Árvore, 26(6): 655664. doi: 10.1590/S0100-67622002000600002

Grigio, M.L. 2013. Caracterização e conservação pós-colheita de camu-camu (Myrciaria dubia 
(Kunth) Mc Vaugh). Dissertação (Mestrado em Agronomia), Universidade Federal de Roraima, Boa Vista, Brasil, 72pp.

Lima, R.; Fernandes, V. L. B.; Oliveira, V.H.; Hernandez, F. F. F. 2001. Crescimento de mudas de cajueiro-Anão-precoce 'CCP-76' submetidas à adubação orgânica e mineral. Revista Brasileira de Fruticultura, 23(2): 391-395.

Malavolta, E; Romero, J.P. 1975. Manual da adubação. 2 ed. São Paulo, ANDA. 346 pp.

Marcussi, F.F.N.; Godoy, L.J.G.; Bôas, R.L.V. 2004. Fertirrigação nitrogenada e potássica na cultura do pimentão baseada no acúmulo de $\mathrm{N}$ e $\mathrm{K}$ pela planta. Irriga, 9(1): 41-51.

Mendonça, V.; Ramos, J.D.; Abreu, N.A.A.; Teixeira, G.A.; Souza, H.A.; Gurgel, R.L.S.; Orbes, M.Y. 2009. Adubação nitrogenada em cobertura na formação e substratos na formação de mudas de mamoeiro 'Formosa'. Ciência e Agrotecnologia, 33(3): 668-675. doi: 10.1590/S1413-70542009000300002

Natale, W.; Prado, R.M.; Almeida, E.V.; Barbosa, J.C. 2006. Adubação nitrogenada e potássica no estado nutricional de mudas de maracujazeiroamarelo. Acta Scientiarum Agronomy, 28(2): 187-192. doi: 10.4025/actasciagron.v28i2.1036

Pinto, J.M.; Soares, J.M.; Choudhury, E.N.; Pereira, J.R. 1994. Efeitos de períodos e de freqüências da fertirrigação nitrogenada na produção do melão. Pesquisa Agropecuária Brasileira, 29(9): 1345-1350.

Ribeiro, S.I.; Mota, M.; Padinha, M. 2002. Recomendações para o cultivo do Camucamuzeiro no Estado do Pará. Circular Técnica, 31: 1-9. (http://www.biblioteca florestal.ufv.br/bitstream/handle/123456789/74 52/ $\operatorname{circtec} 31$.pdf? sequence $=1$ ). Acceso: 20/02/2012

Rodrigues, T.E.; Gama, J.R.N.F.; Rego, R.S.; Lima, A.A.C.; Silva, J.M.L.; Barreto, W.O. 2000. Caracterização e classificação dos solos do Campo Experimental de Água Boa - Embrapa Roraima, Boa Vista, RR. Belém: Embrapa Amazônia Oriental, 43pp.

Serrano, L.A.L.; Marinho, C.S.; Carvalho, A.J.C.; Monnerat, P.H.2004. Efeito de sistemas de produção e doses de adubo de liberação lenta no estado nutricional de porta-enxerto cítrico. Revista Brasileira de Fruticultura, 26(3): 524528.

Silveira, R.L.V.A.; Malavolta, E. 2006. Nutrição e adubação potásica em Eucalyptus. Informaçônes Agronômicas, 71: 1-12. (http://www.ipni.net/publication/ia-
brasil.nsf/0/7852024C3430C60383257AA3006 96DCD/\$FILE/Encarte\%2091.pdf). Acesso 29/11/2013.

Soares, I.; Lima, S.C.; Crisóstomo, L.A. 2007. Crescimento e composição mineral de mudas de gravioleira em resposta a doses de fósforo. Revista Ciência Agronômica, 38(4): 343-349.

Souza, A.S.; Távora, F.J.A.F.; Pitombeira, J.B.; Bezerra, F.M.L. 2007. Épocas de plantio e manejo da irrigação para a mamoneira. II Crescimento e produtividade. Revista Ciência Agronômica, 38(4): 422-429.

Teixeira, A.H.C.; Bassoi, L.H.; Reis, V.C.S.; Silva, T.G.; Ferreira, M.N.L., MAIA, J.L.T 2003.

Estimativa do consumo hídrico da goiabeira, utilizando estações agrometeorológicas automática e convencional. Revista Brasileira de Fruticultura, 25(3): 457-460. doi: 10.1590/S0100-29452003000300024

Teixeira, L.A.J.; Natale, W.; Martins, A.L.M. 2007. Nitrogênio e potássio via fertirrigação e adubação convencional - estado nutricional das bananeiras e produção de frutos. Revista Brasileira de Fruticultura, 29(1): 153-160. doi: 10.1590/S0100-29452007000100032

Viana, M. E. 2007. Interação de nitrogênio e potássio na nutrição, no teor de clorofila e na atividade da redutase do nitrato em plantas de trigo. Dissertação (Mestrado em Agronomia), Escola Superior de Agricultura Luiz de Queiroz, Piracicaba, Brasil. 96pp.

Welter, M.K.; Melo, V.F.; Bruckner, C.H.; Góes, H.T.P.; Chagas, E. A.; Uchôa, S.C.P. 2011. Efeito da aplicação de pó de basalto no desenvolvimento inicial de mudas de camucamu (Myrciaria dubia H.B.K. McVaugh) Revista Brasileira de Fruticultura, 33(3): 922931. doi: 10.1590/S0100-29452011000300028

Yuyama, K.; Aguiar, J.P.L.; Yuyama, L.K.O. 2002. Camu-camu: um fruto fantástico como fonte de vitamina C. Acta Amazônica, 32(1): 169-174. (https://acta.inpa.gov.br/fasciculos/321/PDF/v32n1a16.pdf). Acesso: 12/05/2012

Yuyama, K.; Mendes, N.B.; Valente, J.P. 2011. Longevidade de sementes de camu-camu submetidas a diferentes ambientes e formas de conservação. Revista Brasileira de Fruticultura, 33(2): 601-607. doi: 10.1590/S010029452011005000067

\section{Recibido: 23/01/2014}

Aceptado para publicación: 25/04/2014 